\title{
Calidad de vida de los adultos mayores con sedentarismo del núcleo Xochitlán, Progreso de Obregón Hidalgo
}

\author{
Quality of Life of Older Adults with Sedentarism of the nucleo Xochitlan, Progress of Obregon \\ Hidalgo
}

\author{
Briseyda I. Joaquín-Calva ${ }^{a}$, Ariana. Maya-Sánchez ${ }^{b}$, Benjamín. López-Nolasco ${ }^{c}$, Gabriela \\ Maldonado-Muñiz ${ }^{d}$
}

\begin{abstract}
:
Introduction: Quality of life is a state of general satisfaction and a subjective feeling of physical, psychological and social well-being. However, sedentary older adults have a deficit in their self-care, which is implicated in their quality of life. Objective: know the quality of life of the elderly with sedentary lifestyle of the Xochitlán nucleus, Progress of Obregón Hidalgo. Methodology: Basic applicability research, a quantitative approach with a descriptive scope level, with a non-experimental, cross-sectional and retrospective type design, with a sample of 13 participants, the WHOQOL-BREF instrument was applied with a Cronbach alpha coefficient of 0.70 . Result: Most of the population is 65 years old, with a result of $23.1 \%, 100 \%$ belongs to the female gender and for chronic diseases that older adults present, $61,5 \%$ belongs to hypertension, $15.4 \%$ is attributed to diabetes mellitus and $23.1 \%$ to the absence of disease, in terms of quality of life $100 \%$ of the population has a regular quality of life. Conclusions: The older adults studied have a regular quality of life due to the fact that they become sedentary, and this has an unfavorable impact on the quality of life.
\end{abstract}

Keywords:

Quality of life, older adults, sedentarism.

\begin{abstract}
Resumen:
Introducción: La calidad de vida es un estado de satisfacción general y una sensación subjetiva de bienestar físico, psicológico y social. Sin embargo, los adultos mayores sedentarios presentan un déficit en su autocuidado lo cual se ve implicado en la calidad de vida. Objetivo: Conocer la calidad de vida de los adultos mayores con sedentarismo del núcleo Xochitlán, Progreso de Obregón Hidalgo. Metodología: Aplicabilidad básica, enfoque cuantitativo con un nivel de alcance descriptivo, con diseño de tipo no experimental, de corte transversal y de tipo retrospectivo, con una muestra de 13 participantes, se aplicó el instrumento WHOQOLBREF con un coeficiente de alfa Cronbach de 0.70. Resultados: La mayor parte de la población tiene 65 años de edad, con un resultado de $23,1 \%$, el $100 \%$ pertenece al género femenino y para las enfermedades crónicas que presentan los adultos mayores un $61,5 \%$ pertenece a hipertensión, el $15,4 \%$ se le atribuye a diabetes mellitus y el $23,1 \%$ para la ausencia de enfermedad, en cuanto a la calidad de vida el $100 \%$ de la población tiene una calidad de vida regular. Conclusión: Los adultos mayores estudiados tienen una calidad de vida regular debido a que se vuelven sedentarios, y esto repercute de manera desfavorable en la calidad de vida.
\end{abstract}

Palabras Clave:

Calidad de vida, adulto mayor, sedentarismo

a E.L.E Briseyda Ivette Joaquín Calva, Universidad Autónoma del Estado de Hidalgo, https://orcid.org/ 0000-0002-9355-4082, Email: jo304755@uaeh.edu.mx

b M.C.E Ariana Maya Sánchez, Universidad Autónoma del Estado de Hidalgo, https://orcid.org/0000-0002-6479-2524, Email: ariana_maya8228@uaeh.edu.mx

D.C.E Benjamìn López Nolasco, Universidad Autónoma del Estado de Hidalgo, https://orcid.org/0000-0003-4566-214X, Email: Benjamin_lopez8496@uaeh.edu.mx

d D.C.S Gabriela Maldonado Muñìz, Universidad Autónoma del Estado de Hidalgo, https://orcid.org/0000-0002-4967-1812, Email: gmaldonado@uaeh.edu.mx 


\section{Introducción}

Hoy en día, hay 125 millones de personas con 80 años o más y para el 2050, habrá un número casi igual de personas 434 millones de personas en todo el mundo. La evidencia científica muestra que numerosas enfermedades son más comunes en adultos mayores que tienen poca o nula participación en la actividad física, en comparación con aquellos activos regulares ${ }^{1}$. La Organización Mundial de la Salud señala que al menos un $60 \%$ de la población mundial no realiza la actividad física necesaria para obtener beneficios para la salud. Esto se debe en parte a la insuficiente participación en la actividad física durante el tiempo de ocio y a un aumento de los comportamientos sedentarios durante las actividades laborales y domésticas. El aumento del uso de los medios de transporte "pasivos" también ha reducido la actividad física ${ }^{2}$.

Un estudio realizado por el Instituto Nacional de Estadística y Geografía (INEGI) reveló que porcentaje de población adulta inactiva físicamente es de $57.6 \%^{3}$.

La falta de ejercicio regular es uno de los tantos factores que intervienen en la calidad de vida de los adultos mayores. Hoy la actualidad, la calidad de vida se define como un constructo complejo, multifactorial, en donde se acepta que hay una dualidad subjetiva-objetiva ${ }^{4}$. Abarca múltiples dimensiones, incluidas la física, funcional, social y bienestar psicológico. Haciendo énfasis en la actividad física, esta tiene múltiples beneficios como son la funcionalidad de todos los órganos y sistemas, activa las funciones cerebrales, mejora la flexibilidad, fuerza, coordinación y equilibrio entre algunas otras ventajas, sin embargo, con el paso de los años, los adultos mayores dejan de practicar ciertas actividades, ya que se encuentran limitados físicamente y por lo tanto se vuelven inactivos ${ }^{5}$. Se observa que algunos adultos mayores suelen presentar una dificultad de aceptación y adaptación a las nuevas situaciones originarias del proceso natural de envejecimiento, motivo por el cual muchos de ellos tienen dificultad para llevar una vida saludable, independiente y con autonomía ${ }^{6}$.

El objetivo del presente estudio es conocer la calidad de vida de los adultos mayores con sedentarismo del núcleo Xochitlán, Progreso de Obregón Hidalgo.

\section{Materiales y métodos.}

El presente trabajo de investigación es de aplicabilidad básica con un enfoque cuantitativo. El diseño es de tipo no experimental y de tipo retrospectivo y cuenta con un nivel de alcance descriptivo.

El tipo de muestreo que se utilizó es no probabilístico con una muestra por conveniencia de las cuales se tomó de acuerdo a los siguientes criterios de inclusión:

1. Adultos mayores de 65 años.

2. Pertenecientes al núcleo Xochitlán, Progreso de Obregón Hidalgo que presenten sedentarismo.

3. Ambos sexos.

4. Adultos mayores sedentarios que estén de acuerdo en colaborar con la investigación.

5. Adultos mayores que firmen el consentimiento informado.

Extrayendo así del universo, 13 adultos mayores que participaron en la investigación. La muestra perteneció al núcleo Xochitlán, Progreso de Obregón Hidalgo.

Los criterios de exclusión fueron:

1. Adultos menores de 65 años.

2. Adultos mayores activos físicamente.

3. Adultos mayores sin disponibilidad para apoyar en dicha investigación.

4. Adultos mayores que no deseen firmar el consentimiento informado.

Se aplicó el instrumento WHOQOL-BREF en su versión corta, el cual captura muchos aspectos subjetivos de la calidad de vida.

El instrumento está compuesto por 26 preguntas, de las cuales, una inquiere sobre la calidad de 
vida general, una sobre satisfacción con la salud y los 24 restantes se agrupan en cuatro dominios de calidad de vida, siendo esta salud física (7 preguntas), salud psicológica (6 preguntas), relaciones sociales (3 preguntas) y ambiente (8 preguntas).

Cada encuestado debe responder a cada faceta en categorías cuyo puntaje oscila entre 1 y 5 puntos (Escala Likert). Los puntajes se clasifican en:

- $\quad 1$ a 43 puntos = Calidad de vida mala.

- 44 a 86 puntos = Calidad de vida regular

- $\quad 87$ a 130 puntos = Calidad de vida buena

Se considera que a mayor puntuación mejor $\mathrm{CV}^{7}$.

La confiabilidad del instrumento es de un coeficiente de alfa Cronbach de 0.70 para todas las dimensiones y un $\alpha=.895$ global.

En México, la aplicación del WHOQOL-BREF se realizó a través de un proceso de traducción, retraducción y adaptación al lenguaje de uso común mediante una evaluación de los ítems por 21 jueces expertos, encontrándose un acuerdo entre los ítems de $69.05 \%$ para dar validez de contenido ${ }^{8}$.

Para las variables sociodemográficas se tomaron en cuenta la edad, peso, género y enfermedad crónica. Posterior a la recolección de datos y para su análisis se utilizó el paquete estadístico SPSS en su versión 25 .

\section{Resultados}

El presente estudio se realizó en el núcleo Xochitlán, Progreso de Obregón Hidalgo, se dio inicio en febrero del 2020.

En las siguientes tablas de frecuencias $y$ porcentajes se exponen los resultados de las variables sociodemográficas utilizadas en esta investigación, como lo son: Edad, peso, género y enfermedades crónicas.

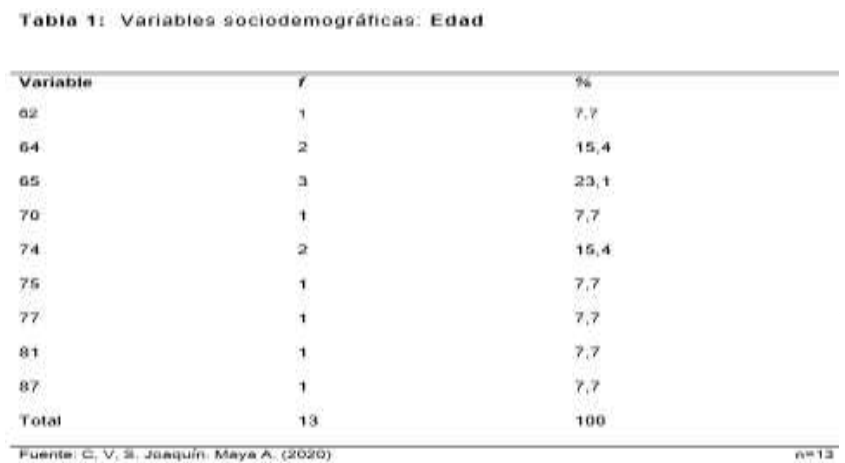

distribuidos en rangos diterentes de edad a partir de los 62 años, la mayoría de estos resultan con un $7,7 \%$.

\begin{tabular}{lll} 
Tabla 2: Variables sociodemográficas: Peso & & $\%$ \\
\hline Variable & $f$ & 7,7 \\
54,00 & 1 & 7,7 \\
57,00 & 1 & 7,7 \\
60,00 & 1 & 7,7 \\
61,00 & 1 & 15,4 \\
62,00 & 2 & 15,4 \\
68,00 & 2 & 7,7 \\
72,00 & 1 & 7,7 \\
74,00 & 1 & 7,7 \\
76,00 & 1 & 7,7 \\
79,00 & 1 & 7,7 \\
85,00 & 1 & $n=13$ \\
\hline Fuente: C. V. S. Joaquin, B. Mays A. (2020) & &
\end{tabular}

En la tabla 2, se indica el peso corporal de los adultos mayores, de los cuales cuatro de ellos mostraron prevalencia entre $62,00 \mathrm{~kg}$ y $68,00 \mathrm{~kg}$ obteniendo así un $15,4 \%$, de igual manera se observa que los nueve adultos restantes tienen un peso diferente a los demás, cada uno de ellos dando resultado del $7,7 \%$.

\section{Tabla 3. Variakles sociddenoyáficas Género}

\begin{tabular}{lcc}
\hline Variable & $f$ & $\%$ \\
Mujer & 13 & 100 \\
\hline Fuente C, V, S. Losaunin, B. Maga, A. (2000) & & $n=13$
\end{tabular}

La presente tabla 3 expone el género de los 13 adultos mayores, donde observamos que el $100 \%$ pertenece al género femenino. 


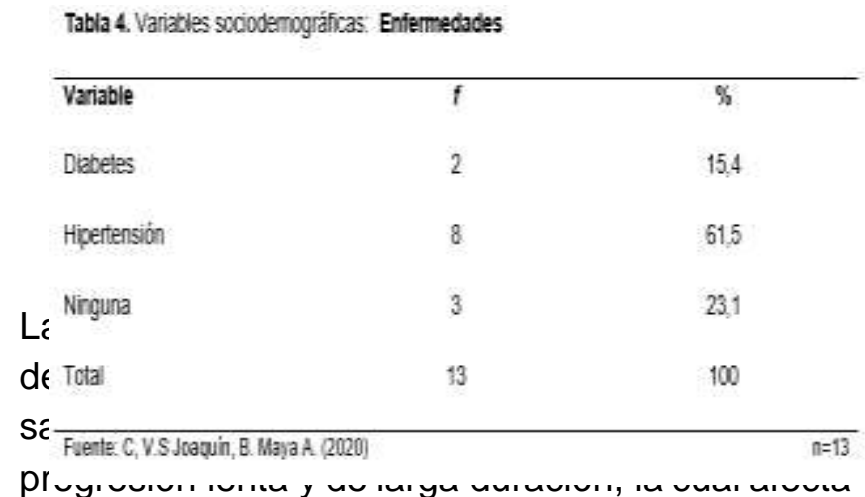

considerablemente a la calidad de vida de las personas, viéndose involucrada la salud, afectando principalmente al sistema cardiovascular, y en muchas ocasiones esto limita la actividad física en la edad longeva. Dando un porcentaje del $61,5 \%$ de adultos mayores hipertensos. También mencionando que el 15,4\% de los adultos mayores presenta diabetes y el $23,1 \%$ no tiene ninguna enfermedad.

En la siguiente tabla de frecuencias y porcentajes se exponen los resultados de la variable independiente utilizada en esta investigación, como lo es, calidad de vida.

\section{Tabla 5: Puntaje total de calidad de vida.}

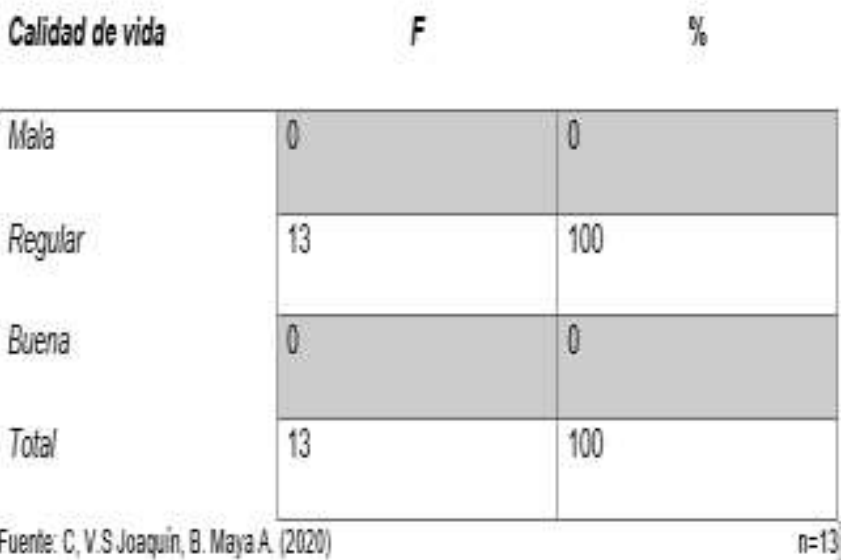

En la tabla 5 se muestran los resultados obtenidos del instrumento WHOQOL-BREF en su versión corta. presentando las frecuencias y porcentajes obtenidas que abarcan los cuatro dominios de calidad de vida, siendo estos salud física, salud psicológica, relaciones sociales y ambiente, así como de las dos primeras preguntas sobre la calidad de vida general $y$ sobre satisfacción con la salud. obteniendo que el $100 \%$ de los participantes se encuentran en una calidad de vida regular ya que sus puntajes oscilan de 44 a 86 puntos, descartando con un $0 \%$ las posibilidades de tener una mala o buena calidad de vida; de esta manera se aprueba la hipótesis de trabajo.

\section{Discusión}

Arocha Rodulfo J. en su artículo "Sedentarismo, la enfermedad del siglo XXl" señala que el sedentarismo y la inactividad física son altamente prevalentes globalmente y están asociados a un amplio rango de enfermedades crónicas y muerte prematura. el interés en la conducta sedentaria está justificado por la creciente evidencia que apunta hacia una relación entre esta conducta y el incremento en la prevalencia de obesidad, diabetes y enfermedad cardiovascular. a través de la historia es bien conocido que ser inactivo no es saludable, pero hoy día casi un tercio de la población del mundo es inactiva, lo que representa un serio problema de salud pública ${ }^{9}$.

Por otro lado, M.T. Loredo-Figueroa y colaboradores en su estudio "Nivel de dependencia, autocuidado y calidad de vida del adulto mayor" encuestaron a 116 adultos mayores obteniendo en la calificación global de las actividades de autocuidado el $22.4 \%$ tuvieron buena capacidad y el $76.6 \%$, muy buena capacidad. en la percepción de calidad de vida en la dimensión física, el $24 \%$ de los entrevistados consideran que su salud es buena y el $56 \%$, regular. en las pruebas de correlación se encontró mínima relación entre las variables de autocuidado y dependencia; escasa entre autocuidado y grado de escolaridad; correlación positiva entre la dependencia y la calidad de vida, así como la calidad de vida y la edad, aunque el valor de todas las correlaciones fue bajo el $24 \%$ de los entrevistados consideran que su salud es buena y el $56 \%$, regular. de manera tal que, a menor dependencia y mayor autocuidado, mayor es la calidad de vida del adulto mayor ${ }^{10}$.

La calidad de vida se relaciona a factores económicos, sociales y culturales en el entorno 
que se desarrolla y, en gran medida, a la escala de valores de cada individuo según el autor Pinedo Varela en su artículo titulado "Salud y calidad de vida en el adulto mayor" publicado en abril-junio del 2016 en Lima Perú por tanto, se consensó en que la calidad de vida deber ser considerada desde una visión multidimensional, que además de lo anteriormente mencionado, debe incluir aspectos ambientales, culturales, económicos, de servicios de salud, satisfacción, actividades de ocio, entre otros ${ }^{11}$.

En el presente trabajo de investigación titulado "Calidad de vida de los adultos mayores con sedentarismo del núcleo Xochitlán, Progreso de Obregón Hidalgo" se logró determinar la calidad de vida de los adultos mayores sedentarios y teniendo en consideración los estudios previos, coincidimos en que los adultos mayores tienen una mejor calidad de vida al ser personas activas, y que el sedentarismo se asocia con una gran cantidad de padecimientos funcionales, esto quiere decir que, al desarrollar una enfermedad crónica debido a un estilo de vida sedentario, la calidad de vida del adulto mayor disminuye.

\section{Conclusión}

Se concluye que la calidad de vida de los adultos mayores fue consistentemente inferior al nivel óptimo postulado por la encuesta WHOQOLBREF, obteniendo una calidad de vida regular, oscilando puntajes de 44 a 86 puntos respectivamente, esto debido a que se vuelven sedentarios. Optar por un estilo de vida sedentario repercute de manera desfavorable en la calidad de vida, ya que el sedentarismo es un factor de riesgo para desarrollar enfermedades crónicas trayendo consigo una disminución en la calidad de vida del adulto mayor.

Destacamos que un estilo de vida inactivo también puede aumentar el riesgo de muerte prematura y cuanto más sedentario sea, mayor es el riesgo para la salud ${ }^{12}$.

\section{Recomendaciones}

La calidad de vida de los adultos mayores consiste en un sistema complejo, porque envuelve la relación de factores internos y externos al individuo como, por ejemplo, la subjetividad y el contexto sociocultural. Algunas recomendaciones son:

- Guiar o implementar acciones que podrían ayudar a mejorar conductas 0 estilos de vida.

- El personal de enfermería es capaz de transformar estilos de vida inadecuados, en este caso, podría favorecer el autocuidado de los adultos mayores sedentarios implementando el apoyoeducación.

- Aumentar la prevención y acrecentar la capacidad de hacer frente a nuevas conductas generadoras de salud tales como el autocuidado.

- La ayuda mutua y facilitar ambientes saludables, ya que evidentemente la presencia del sedentarismo en este grupo etario compromete el bienestar y satisfacción en la calidad de vida.

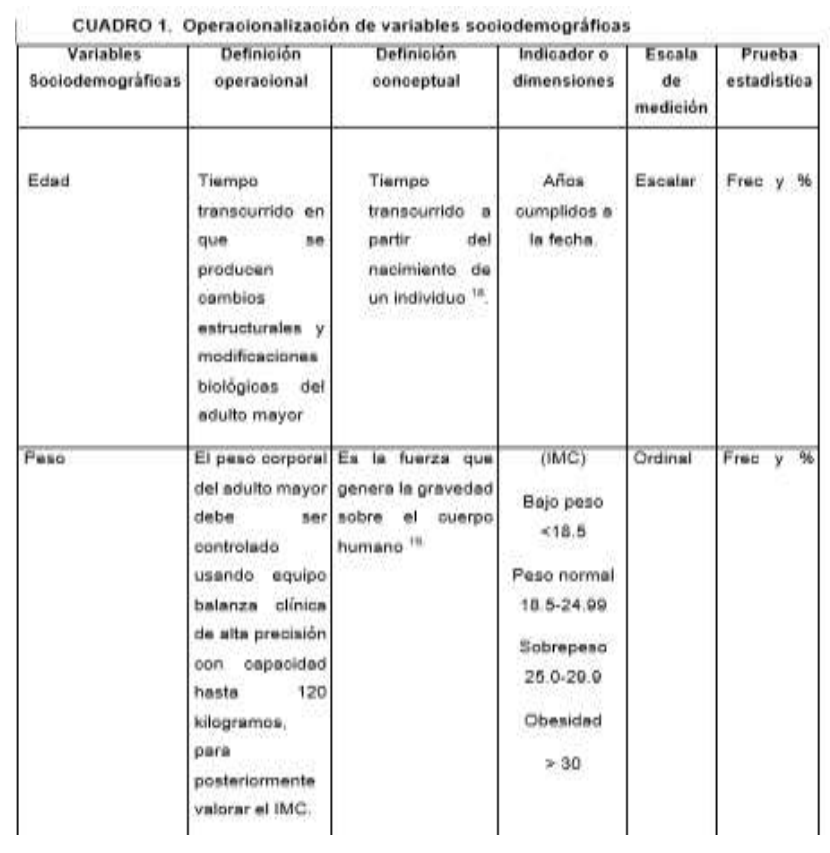




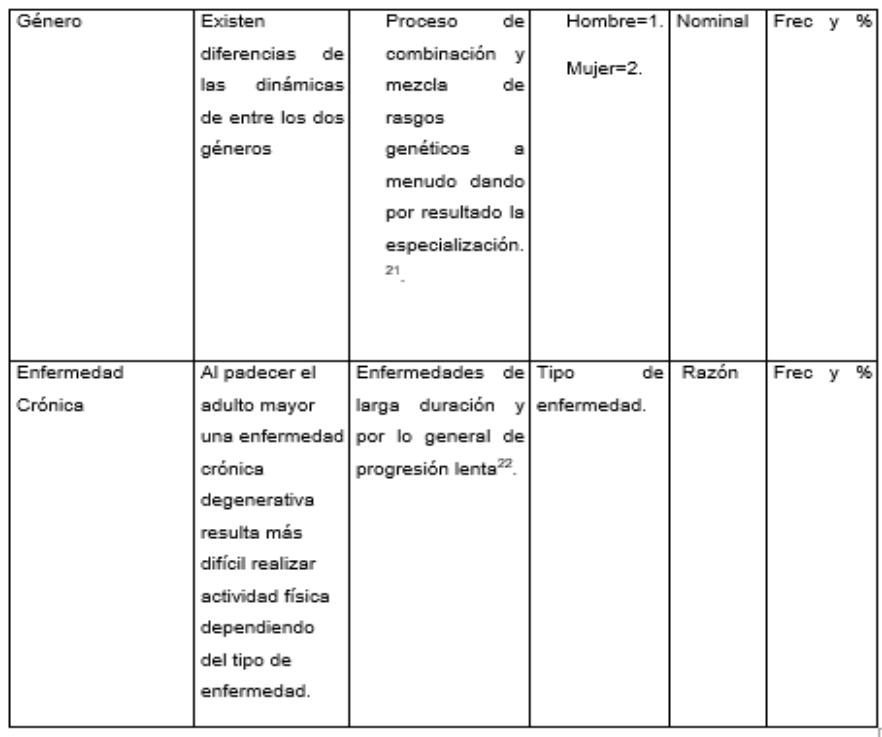

CUADRO 2. VARIABLE INDEPENDIENTE

\begin{tabular}{|c|c|c|c|c|c|}
\hline $\begin{array}{c}\text { Variable } \\
\text { Dependiente }\end{array}$ & $\begin{array}{c}\text { Definición } \\
\text { operacional }\end{array}$ & $\begin{array}{l}\text { Definición } \\
\text { conceptual }\end{array}$ & $\begin{array}{l}\text { Indicador o } \\
\text { dimensiones }\end{array}$ & $\begin{array}{l}\text { Escala de } \\
\text { medición }\end{array}$ & $\begin{array}{c}\text { Prueba } \\
\text { estadística }\end{array}$ \\
\hline $\begin{array}{l}\text { Calidad de } \\
\text { vida }\end{array}$ & $\begin{array}{l}\text { La calidad de } \\
\text { vida es un } \\
\text { concepto } \\
\text { utilizado para } \\
\text { evaluar el } \\
\text { bienestar } \\
\text { social general } \\
\text { de } \\
\text { individuos y } \\
\text { sociedades } \\
\text { por } \\
\text { mismá es } \\
\text { decir. } \\
\text { informalmente } \\
\text { la calidad de } \\
\text { vida es el } \\
\text { grado en que } \\
\text { los individuos } \\
\text { o sociedades } \\
\text { tienen altos } \\
\text { valores en los } \\
\text { índices de } \\
\text { bienestar } \\
\text { social. }\end{array}$ &  & $\begin{array}{l}\text { 1. Calidad } \\
\text { de vida } \\
\text { buena = } \\
87-130 \\
\text { puntos } \\
\text { 2. Calidad } \\
\text { de vida } \\
\text { regular = } \\
44-86 \\
\text { puntos. } \\
\text { 3. Calidad } \\
\text { de vida } \\
\text { mala = 1- } \\
43 \\
\text { puntos. }\end{array}$ & $\begin{array}{l}\text { Instrumento } \\
\text { WHOCOL- } \\
\text { BREF para } \\
\text { la medición } \\
\text { de la calidad } \\
\text { de vide. }\end{array}$ & $\begin{array}{l}\text { Frec y \% } \\
\text { Correlación } \\
\text { de Pearson }\end{array}$ \\
\hline
\end{tabular}

\section{CUADRO 3. Intrumento de mediciòn}

\section{WHOQOL-BREF}

Instrucciones: Este cuestionario sirve para conocer su opinión acerca de su calidad de vida, su salud y otras áreas de su vida. Por favor conteste todas las preguntas. Si no está segurola de qué respuesta dar a una pregunta, escoja la que le parezca más apropiada. A veces, esta puede ser la primera respuesta que le viene a la cabeza.

Tenga presente su modo de vivir, expectativas, placeres y preocupaciones. Le pedimos que piense en su vida durante las dos últimas semanas Por ejemplo, pensando en las dos úlimas semanas, se puede preguntar:

\begin{tabular}{|l|l|c|c|c|c|c|}
\hline & Nada & Un poco & Moderado & Bastante & Totalmente \\
\hline $\begin{array}{l}\text { ¿Obtiene de otras personas } \\
\text { el apoyo que necesita? }\end{array}$ & 1 & 2 & 3 & 4 & 5 \\
\hline
\end{tabular}

Rodee con un circulo el nùmero que mejor defina cuánto apoyo obtuvo de otras personas en las dos ultimas semanas. Si piensa que obtuvo bastante apoyo de otras personas, usted deberia seflalar con un circulo el nùmero 4, quedando la respuesta de la siguiente forma:

\begin{tabular}{|l|c|c|c|c|c|c|}
\hline & Nada & Un poco & Moderado & Bastante & Totaimente \\
\hline $\begin{array}{l}\text { ¿Obtiene de otras personas } \\
\text { el apoyo que necesita? }\end{array}$ & 1 & 2 & 3 & 4 & 5 \\
\hline
\end{tabular}

Recuerde que cualquier número es valido, lo importante es que represente su opinión

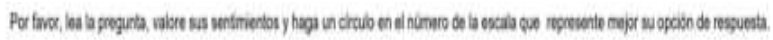

\begin{tabular}{|c|c|c|c|c|c|c|}
\hline & & Moyma & Regiar & Noma & Eaturifixy & Wy biena \\
\hline 1 & ¿Como callerais s caldad de vida? & 1 & 2 & 3 & 4 & 5 \\
\hline & & $\begin{array}{c}\text { My } \\
\text { natstemola }\end{array}$ & $\begin{array}{l}\text { Unpowo } \\
\text { insatsletera }\end{array}$ & Lomemal & $\begin{array}{l}\text { Bostato } \\
\text { entadectoia }\end{array}$ & May sablichola \\
\hline 2 & 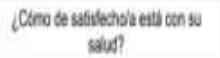 & 1 & 2 & 3 & 4 & 5 \\
\hline
\end{tabular}

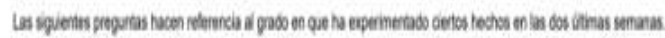

\begin{tabular}{|c|c|c|c|c|c|c|}
\hline & & Nada & Unpose & Lonemal & Bastate & Entromadareite \\
\hline 3 & 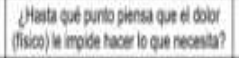 & 1 & 2 & 3 & 4 & 5 \\
\hline 4 &  & 1 & 2 & 1 & 4 & 5 \\
\hline 5 & ¿Curto detra of la vids? & 1 & 2 & 3 & 4 & 5 \\
\hline 6 &  & 1 & 2 & 3 & 4 & 8 \\
\hline 1 & $\begin{array}{l}\text { ¿Oul es wa tapasado of } \\
\text { concintasion? }\end{array}$ & 1 & 2 & 3 & 4 & 8 \\
\hline 8 & 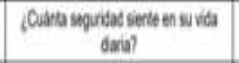 & 1 & 2 & 3 & 4 & 8 \\
\hline 9 & 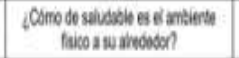 & 1 & 2 & 3 & 4 & 8 \\
\hline
\end{tabular}

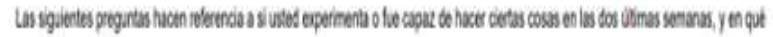

\begin{tabular}{|c|c|c|c|c|c|c|}
\hline & & Nada & Unposo & Lo nomal & Bastare & Totaimecte \\
\hline 10 & 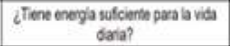 & 1 & 2 & 3 & 4 & 5 \\
\hline 11 & $\begin{array}{l}\text { ¿Es capaz de accotar sw apriencia } \\
\text { fusca? }\end{array}$ & 1 & 2 & 3 & 4 & 5 \\
\hline 12 & 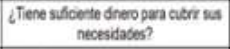 & 1 & 2 & 3 & 4 & 5 \\
\hline 13 & $\begin{array}{l}\text { ¿Dispone de bi infomacion qua } \\
\text { necsta pra su vida diaria? }\end{array}$ & 1 & 2 & 3 & 4 & 5 \\
\hline 14 & 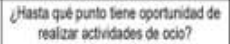 & 1 & 2 & 3 & 4 & 5 \\
\hline 15 & $\begin{array}{l}\text { ¿Es capouz de desplasarse de un bigax } \\
\text { actoo? }\end{array}$ & 1 & 2 & 3 & 4 & 5 \\
\hline
\end{tabular}




\begin{tabular}{|c|c|c|c|c|c|c|}
\hline & & $\begin{array}{c}\text { My } \\
\text { matstectora }\end{array}$ & Poco & Lo nomul & $\begin{array}{l}\text { Bastumita } \\
\text { sastectoda }\end{array}$ & Mey satistectols \\
\hline 16 & $\begin{array}{l}\text { ¿Como de stastectios esth con so } \\
\text { suete? }\end{array}$ & $t$ & 2 & 3 & 4 & s. \\
\hline 17 &  & 1 & 2 & 3 & 4 & 5 \\
\hline 13 & 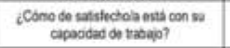 & 1 & 2 & 3 & 4 & 5 \\
\hline 19 & $\begin{array}{l}\text { ¿Como de sesclectiolie enta de sil } \\
\text { mismo? }\end{array}$ & 1 & 2 & 3 & 4 & 5 \\
\hline 20 & 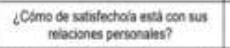 & 1 & 2 & 3 & 4 & 5 \\
\hline 21 &  & 1 & 2 & 3 & 4 & 5 \\
\hline 2 & 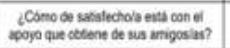 & 1 & 2 & 3 & 4 & 5 \\
\hline 23 & 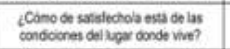 & 1 & 2 & 3 & 4 & 5 \\
\hline 24 & 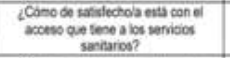 & 1 & 2 & 3 & 4 & 5 \\
\hline s &  & 1 & 2 & 3 & 4 & 5 \\
\hline
\end{tabular}

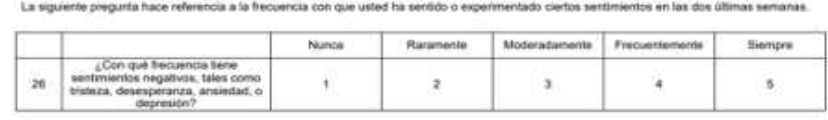

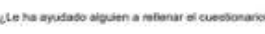





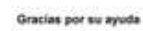

\section{Referencias}

1.Acosta Quiroz, C. O., Vales Garcìa, J. J., Echeverrìa Castro, S. B., Serrano Encinas, D. M., \& Garcìa Flores, R. (Julio-diciembre de 2013). Obtenido de Confiabilidad y validez del Cuestionario de Calidad de Vida (WHOQOLOLD) en adultos mayores mexicanos: https://www.uv.mx/psicysalud/psicysalud-23-2/23-

2/Christian\%20Oswaldo\%20Acosta\%20Quiroz.pdf

2. Aponte Daza, V. C. (Agosto de 2015). Scielo. Obtenido de CALIDAD DE VIDA EN LA TERCERA EDAD: http://www.scielo.org.bo/scielo.php?script=sci_art text\&pid=S2077-21612015000200003

3.INEGI. (2017). El economista. Obtenido de https://www.eleconomista.com.mx/deportes/Meno s-de-la-mitad-de-mexicanos-hace-ejercicio-segunel-Inegi-20180126-0056.html

4.Menèndez Montañès , M. C., Brochier , K., \& Bernardete, R. (2013). c.La actividad física y la psicomotricidad en las personas mayores: sus contribuciones para el envejecimiento activo, saludable y satisfactorio. Obtenido de https://www.redalyc.org/pdf/3215/321527168014. pdf

5.OMS. (2020). Inactividad física: un problema de salud pública mundial. Obtenido de https://www.who.int/dietphysicalactivity/factsheet inactivity/es/\#: :text=Al\%20menos\%20un\%206 $0 \% 25 \% 20$ de,las $\% 20$ actividades $\% 20$ laborales $\% 20$ y\%20dom\%C3\%A9sticas.
6.Organizacion Mundial de la Salud.(2020). Envejecimiento y salud.Obtenido de https://www.who.int/es/newsroom/fact-sheets/detail/envejecimiento-ysalud\#: :text=Hoy $\% 20 \mathrm{en} \% 20 \mathrm{~d} \% \mathrm{C} 3 \% \mathrm{ADa} \% 2 \mathrm{C} \%$ 20hay $\% 20125$,de $\% 20$ ingresos $\% 20$ bajos $\% 20$ y $\% 20$ medianos.

7.Salud, M. d. (2015). Guia de actividad fisica. Obtenido de https://www.paho.org/uru/index.php?option=com docman\&view=download\&slug=guia-deactividad-fisica-msp-compressed\&Itemid $=307$

8.Urzùa M, A., \& Caqueo Urizar, A. (2013). Estructura Factorial y valores de referencia del Wholqol Bref en poblaciòn adulta chilena. Obtenido de Scielo: https://scielo.conicyt.cl/pdf/rmc/v141n12/art08.pd $\mathrm{f}$

9.Arocha Rodulfo, J. (Octubre de 2019). Sedentarismo, la enfermedad del siglo xxi. Obtenido de https://www.sciencedirect.com/science/article/abs/pii/S021 $\underline{491681930054}$

10.Loredo Figueroa, M. T., Gallegos Torres, R., Xeque Morales, A., Palomè Vega, G., \& Juàrez Lira, A. (2016). ENFERMERIA UNIVERSITARIA Nivel de dependencia, autocuidado y calidad de vida. Obtenido de http://www.scielo.org.mx/pdf/eu/v13n3/1665-7063-eu-13-

11.Varela Pinedo, L. (2016). SCIELO Salud y calidad de vida en el adulto mayor. Obtenido de http://www.scielo.org.pe/scielo.php?script=sci arttext\&pid $=\mathrm{S} 17$

12.S, M. (2016). Sedentarismo y salud. Obtenido de http://madridsalud.es/

13.Mèdico, D. (2015). Clinica Universidad de Navarra. Obtenido de https://www.cun.es/diccionario$\underline{\text { medico/terminos/edad }}$

14.Acero, J. (2015). G-SE Peso Corporal. Obtenido de https://g-se.com/peso-corporal-bp-

857cfb26e59136\#: :text=El\%20peso\%20corporal\%20es\% 201a,factor\%20aceleraci\%C3\%B3n\%20de\%20la\%20grave dad.

15.Rivera, A. (Enero de 2015). Biologìa. Obtenido de https://prezi.com/okpariah-ryc/en-biologia-el-sexo-es-unproceso-de-combinacion-y-mezcla-d/\#: :text=Sexo-

,En\%20biolog\%C3\%ADa\%2C\%20e1\%20sexo\%20es\%20u n\%20proceso \%20de\%20combinaci\%C3\%B3n\%20y,mascu lina $\% 20$ (conocidas $\% 20$ como $\% 20$ s

16.Salud, O. M. (Marzo de 2015). Obtenido de OMS: https://www.who.int/topics/chronic_diseases/es/\#: :text=L as $\% 20$ enfermedades $\% 20 \mathrm{cr} \% \mathrm{C} 3 \%$ B3nicas $\% 20$ son $\% 20$ enfer medades,del $\% 2063 \% 25 \% 20 \mathrm{de} \% 201$ las $\% 20$ muertes.

17.Ardila, R. (2015). Calidad de vida: una definición integradora.

Obtenido

de 
Publicación semestral, XIKUA Boletín Científico de la Escuela Superior de Tlahuelilpan, Vol. 9, No. 17 (2021) 18-25

https://www.researchgate.net/publication/26595141_Calida d_de_vida_una_definici 